International Journal of Pure and Applied Mathematics

Volume 115 No. 2 2017, 353-360

ISSN: 1311-8080 (printed version); ISSN: 1314-3395 (on-line version)

url: http://www.ijpam.eu

doi: 10.12732/ijpam.v115i2.12

ijpam.eu

\title{
ON AN EXTENSION OF SHARP DOMAINS
}

\author{
Chahn Yong Jung ${ }^{1}$, Waseem Khalid ${ }^{2}$, Waqas Nazeer ${ }^{3}$, \\ Tayyab Tariq ${ }^{4}$, Shin Min Kang ${ }^{5} \S$ \\ ${ }^{1}$ Department of Business Administration \\ Gyeongsang National University \\ Jinju, 52828, KOREA \\ ${ }^{2,4}$ Department of Mathematics \\ The University of Lahore \\ Pakpattan, 57400, PAKISTAN \\ ${ }^{3}$ Division of Science and Technology \\ University of Education \\ Lahore, 54000, PAKISTAN \\ ${ }^{5}$ Department of Mathematics and RINS \\ Gyeongsang National University \\ Jinju 52828, KOREA
}

\begin{abstract}
As an extension of the class of sharp domains introduced by Ahmad et al., we introduce and study a class of integral domains $D$ characterized by the property that whenever $X, Y_{1}, Y_{2}$ are nonzero ideals of $D$ with $X \supseteq Y_{1} Y_{2}$, there exist nonzero ideals $Z_{1}$ and $Z_{2}$ such that $X_{w}=\left(Z_{1} Z_{2}\right)_{w},\left(Z_{1}\right)_{w} \supseteq Y_{1}$ and $\left(Z_{2}\right)_{w} \supseteq Y_{2}$. We call $D$ with this property a $w$-sharp domain. We show that every fraction ring of a $w$-sharp domain is $w$-sharp, that a $w$-Dedekind domain is $w$-sharp and that every nonzero finitely generated ideal of a $w$-sharp domain is $w$-invertible.
\end{abstract}

AMS Subject Classification: 13A15, 13F05

Key Words: Schreier domain, pseudo-Dedekind domain, Prüfer domain

Received: January 31, 2017

Revised: July 5, 2017

Published: July 14, 2017

(c) 2017 Academic Publications, Ltd. url: www.acadpubl.eu

$\S_{\text {Correspondence author }}$ 


\section{Introduction}

In [9], Cohn introduced a Schreier domain. The study of Schreier domains was continued in $[8,19,20]$. Later many extensions of the Schreier domains were introduced and studied in $[1,2,5,10,11,13]$. In [2], Ahmad et al. called a domain $D$ a sharp domain if whenever $X, Y_{1}, Y_{2}$ are nonzero ideals of $D$ with $X \supseteq Y_{1} Y_{2}$, there exist nonzero ideals $Z_{1}$ and $Z_{2}$ such that $X=Z_{1} Z_{2}, Z_{1} \supseteq Y_{1}$ and $Z_{2} \supseteq Y_{2}$. They showed that a sharp domain is a completely integrally closed generalized GCD domain, that a sharp domain is a Prüfer domain of dimension $\leq 1$ and that a countable sharp domain is a Dedekind domain.

In this paper, we study the following extension of a sharp domain. We call a domain $D$ a $w$-sharp domain if whenever $X, Y_{1}, Y_{2}$ are nonzero ideals of $D$ with $X \supseteq Y_{1} Y_{2}$, there exist nonzero ideals $Z_{1}$ and $Z_{2}$ such that $X_{w}=\left(Z_{1} Z_{2}\right)_{w}$, $\left(Z_{1}\right)_{w} \supseteq Y_{1}$ and $\left(Z_{2}\right)_{w} \supseteq Y_{2}$.

We show that every fraction ring of a $w$-sharp domain is $w$-sharp (Proposition 2.3). A $w$-sharp domain is a $\mathrm{P} w \mathrm{MD}$ of $w$-dimension $\leq 1$ (Proposition 2.4). A $w$-Dedekind domain is a $w$-sharp domain (Proposition 2.5). The $v$ closure of every nonzero ideal of a $w$-sharp domain is $w$-invertible (Proposition 2.6). A $w$-sharp Mori domain is $w$-Dedekind (Corollary 2.7). Every nonzero finitely generated ideal of a $w$-sharp domain is $w$-invertible (Proposition 2.10). A countable $w$-sharp domain is a $w$-Dedekind domain (Corollary 2.13).

This new concept of $w$-sharp domain depends upon the notion of star operation, w-operation. A reader in need of a quick review on this topic may consult Section 32 and 34 of [15]. For the reader's convenience we give a working introduction here for the notions involved. Let $D$ be a domain with quotient field $K$ and let $F(D)$ denote the set of nonzero fractional ideals of $D$. A function $A \mapsto A^{*}: F(D) \rightarrow F(D)$ is called a star operation on $D$ if $*$ satisfies the following three conditions for all $0 \neq a \in K$ and for all $I, J \in F(D)$ : (1) $D^{*}=D$ and $(a I)^{*}=a I^{*},(2) I \subseteq I^{*}$ and if $I \subseteq J$, then $I^{*} \subseteq J^{*},(3)$ $\left(I^{*}\right)^{*}=I^{*}$. An ideal $I \in F(D)$ is called a $*$-ideal if $I^{*}=I$. For all $I, J \in F(D)$, we have $(I J)^{*}=\left(I^{*} J\right)^{*}=\left(I^{*} J^{*}\right)^{*}$. These equations define the so-called $*$ multiplication. If $\left\{I_{\alpha}\right\}$ is a subset of $F(D)$ such that $\cap I_{\alpha} \neq 0$, then $\cap I_{\alpha}^{*}$ is a *-ideal. Also, if $\left\{I_{\alpha}\right\}$ is a subset of $F(D)$ such that $\sum I_{\alpha}$ is a fractional ideal, then $\left(\sum I_{\alpha}\right)^{*}=\left(\sum I_{\alpha}^{*}\right)^{*}$. A star operation $*$ is said to be a stable star operation if $(I \cap J)^{*}=I^{*} \cap J^{*}$ for all $I, J \in F(D)$. The function $*_{f}: F(D) \rightarrow F(D)$ given by $I^{*_{f}}=\cup J^{*}$, where $J$ ranges over all nonzero finitely generated sub-ideals of $I$, is also a star operation; $*$ is said to be a star operation of finite character if $*=*_{f}$. Clearly $\left(*_{f}\right)_{f}=*_{f}$. Let $\operatorname{Max}_{*}(D)$ denote the set of maximal $*$-ideals, that is, ideals maximal among proper integral $*$-ideals of $D$. Every 
maximal $*$-ideal is a prime ideal. If $*$ is of finite character, then every proper $*$-ideal is contained in some maximal $*$-ideal, and $*$ is stable if and only if $I^{*}=\cap_{P \in \operatorname{Max}_{*}(D)} I D_{P}$ for all $I \in F(D)$, cf. [3, Corollary 4.2]. A $*$-ideal $I$ is of finite type if $I=\left(a_{1}, \ldots, a_{n}\right)^{*}$ for some $a_{1}, \ldots, a_{n} \in I$. An ideal $I \in F(D)$ is said to be $*$-invertible if $\left(I I^{-1}\right)^{*}=D$, where $I^{-1}=(D: I)=\{x \in K \mid x I \subseteq D\}$. If * is of finite character, then $I$ is $*$-invertible if and only if $I I^{-1}$ is not contained in any maximal $*$-ideal of $D$; in this case $I^{*}=\left(a_{1}, \ldots, a_{n}\right)^{*}$ for some $a_{1}, \ldots, a_{n} \in I$. Let $*_{1}, *_{2}$ be star operations on $D$. We write $*_{1} \leq *_{2}$, if $I^{*_{1}} \subseteq I^{*_{2}}$ for all $I \in F(D)$. In this case we get $\left(I^{*_{1}}\right)^{*_{2}}=I^{*_{2}}=\left(I^{*_{2}}\right)^{*_{1}}$ and every $*_{1}$-invertible ideal is $*_{2}$-invertible. Some well-known star operations are: the $d$-operation (given by $I \mapsto I$ ), the $v$-operation (given by $I \mapsto I_{v}=\left(I^{-1}\right)^{-1}$ ) and the $t$ operation (defined by $t=v_{f}$ ). The $w$-operation is the star operation given by $I \mapsto I_{w}=\{x \in K \mid J x \subseteq I$ for some finitely generated ideal $J$ of $D$ with $\left.J^{-1}=D\right\}$. The $w$-operation is a stable star operation of finite character. For every $I \in F(D)$, we have $I \subseteq I_{w} \subseteq I_{t} \subseteq I_{v}$; so a $v$-ideal is a $t$-ideal and a $t$-ideal is a $w$-ideal. It is known that $\operatorname{Max}_{w}(D)=\operatorname{Max}_{t}(D)$, cf. [4, Corollary 2.17] and $I_{w}=\cap_{P \in \operatorname{Max}_{t}(D)} I D_{P}$ by $[4$, Corollary 2.13]. So, a nonzero fractional ideal is $w$-invertible if and only if it is $t$-invertible. Recall from [7] that $D$ is called a $*$-Dedekind domain if every nonzero ideal of $D$ is $*$-invertible.

Throughout this paper, all rings are commutative and unitary. Our standard references for any undefined notation or terminology are [15] and [18].

\section{Main Results}

Definition 2.1. We call a domain $D$ a $w$-sharp domain if whenever $X, Y_{1}, Y_{2}$ are nonzero ideals of $D$ with $X \supseteq Y_{1} Y_{2}$, there exist nonzero ideals $Z_{1}$ and $Z_{2}$ such that $X_{w}=\left(Z_{1} Z_{2}\right)_{w},\left(Z_{1}\right)_{w} \supseteq Y_{1}$ and $\left(Z_{2}\right)_{w} \supseteq Y_{2}$.

Proposition 2.2. Let $D$ be a domain and $T \subseteq D$ a multiplicative set such that $X_{w} \subseteq\left(X D_{T}\right)_{w}$ for each non zero ideal $X$ of $D$. If $D$ is $w$-sharp, then the fraction ring $D_{T}$ is $w$-sharp.

Proof. Given that $X_{w} \subseteq\left(X D_{T}\right)_{w}$ which is equivalent to $\left(X_{w} D_{T}\right)_{w}=$ $\left(X D_{T}\right)_{w}$. If $X, Y_{1}, Y_{2}$ are nonzero ideals of $D$ with $\left(X D_{T}\right) \supseteq\left(Y_{1} Y_{2} D_{T}\right)$, then $Z=X D_{T} \cap D \supseteq Y_{1} Y_{2}$. Since $D$ is $w$-sharp, there exist nonzero ideals $Z_{1}$ and $Z_{2}$ of $D$ such that $Z_{w}=\left(Z_{1} Z_{2}\right)_{w},\left(Z_{1}\right)_{w} \supseteq Y_{1}$ and $\left(Z_{2}\right)_{w} \supseteq$ $Y_{2}$. As $\left(Y D_{T}\right)_{w}=\left(Y_{w} D_{T}\right)_{w}$ for each nonzero ideal $Y$, we have $\left(X D_{T}\right)_{w}=$ $\left(Z_{w} D_{T}\right)_{w}=\left(\left(Z_{1} Z_{2}\right)_{w} D_{T}\right)_{w}=\left(Z_{1} Z_{2} D_{T}\right)_{w},\left(Z_{1} D_{T}\right)_{w}=\left(\left(Z_{1}\right)_{w} D_{T}\right)_{w} \supseteq Y_{1} D_{T}$ and $\left(Z_{2} D_{T}\right)_{w} \supseteq Y_{2} D_{T}$. 
Proposition 2.3. Every fraction ring of a $w$-sharp domain is w-sharp.

Proof. By Proposition 2.2, it is enough to show that $X_{w} \subseteq\left(X D_{T}\right)_{w}$ for each nonzero ideal $X$ of $D$. If $r \in X_{w}$, then $r Y \subseteq X$ for some nonzero finitely generated ideal $Y$ of $D$ such that $Y_{v}=D$. Hence $\left(Y D_{T}\right)_{v}=D_{T}$ by $[17$, Lemma 3.4] and $r Y D_{T} \subseteq X D_{T}$ implies $r \in\left(X D_{T}\right)_{w}$.

Proposition 2.4. If $D$ is a $w$-sharp domain, then $D_{Q}$ is a valuation domain with value group a complete subgroup of the reals for each $Q \in \operatorname{Max}_{w}(D)$. In particular, $D$ is a $P w M D$ of $w$-dimension $\leq 1$.

Proof. Let $Q$ be a maximal $w$-ideal. If $X$ is a nonzero ideal of $D$, then $X_{w} D_{Q}=X D_{Q}$ by [3, Corollary 4.2]. By Proposition 2.2, applied for $T=D-Q$, we get that $D_{Q}$ is a sharp domain. Now apply [2, Theorem 11]. The "in particular" assertion is clear.

Proposition 2.5. If $D$ is a $w$-Dedekind domain, then $D$ is $w$-sharp.

Proof. Let $X, Y_{1}, Y_{2}$ be nonzero ideals of $D$ such that $X \supseteq Y_{1} Y_{2}$. Take $Z_{1}=X+Y_{1}$ and $Z_{2}=X\left(Z_{1}\right)^{-1}$. Note that $Z_{1} \subseteq D$ and $Y_{1} \subseteq Z_{1}$. Since $\left(Z_{1}\left(Z_{1}\right)^{-1}\right)_{w}=D, X_{w}=\left(Z_{1} Z_{2}\right)_{w}$. From $Y_{2} Z_{1}=Y_{2}\left(Y_{1}+X\right) \subseteq X$, we get $Y_{2} \subseteq\left(Y_{2} Z_{1}\left(Z_{1}\right)^{-1}\right)_{w} \subseteq\left(X\left(Z_{1}\right)^{-1}\right)_{w}=\left(Z_{2}\right)_{w}$.

Proposition 2.6. If $D$ is a $w$-sharp domain, then $X_{v}$ is $w$-invertible for each nonzero ideal $X$ of $D$.

Proof. If $X$ is a nonzero ideal of $D$ and $r \in X-\{0\}$, then $X\left(r X^{-1}\right) \subseteq$ $r D$. Since $D$ is $w$-sharp, there exist nonzero ideals $Z_{1}$ and $Z_{2}$ of $D$ such that $\left(Z_{1}\right)_{w} \supseteq X,\left(Z_{2}\right)_{w} \supseteq r X^{-1}$ and $r D=\left(Z_{1} Z_{2}\right)_{w}$. Hence $Z_{1}$ is $w$-invertible and we get $Z_{1}^{-1}=\left(r^{-1} Z_{2}\right)_{w} \supseteq\left(r r^{-1} X^{-1}\right)_{w}=X^{-1}$, so $\left(Z_{1}\right)_{v} \subseteq X_{v}$. The reverse inclusion follows from $\left(Z_{1}\right)_{w} \supseteq X$. Thus $X_{v}=\left(Z_{1}\right)_{v}$ is $w$-invertible, because $\left(Z_{1}\right)_{w}=\left(Z_{1}\right)_{v}$ since $Z_{1}$ is $w$-invertible.

Corollary 2.7. If $D$ is a $w$-sharp and a Mori domain, then $D$ is $w$ Dedekind.

Proof. By Proposition 2.4, $D$ is a $P w M D$, so $w=t$ by $[14$, Proposition 3.15]. As $D$ is a Mori domain, $w=t=v$. By Proposition 2.6, $D$ is $w$ Dedekind.

Lemma 2.8. Let $D$ be a domain and $X, Z_{1}, Z_{2} \in F(D)$.

(1) If $\left(X+Z_{1}\right)_{w}=D$, then $\left(X \cap Z_{1}\right)_{w}=\left(X Z_{1}\right)_{w}$.

(2) If $X$ is $w$-invertible, then $\left(X\left(Z_{1} \cap Z_{2}\right)\right)_{w}=\left(X Z_{1} \cap X Z_{2}\right)_{w}$. 
Proof. (1) Obviously, $\left(X Z_{1}\right)_{w} \subseteq\left(X \cap Z_{1}\right)_{w}$. Conversly, since $\left(X+Z_{1}\right)_{w}=$ $D$, we have $\left(X \cap Z_{1}\right)_{w}=\left(\left(X \cap Z_{1}\right)\left(X+Z_{1}\right)\right)_{w} \subseteq\left(X Z_{1}\right)_{w}$, thus $\left(X \cap Z_{1}\right)_{w}=$ $\left(X Z_{1}\right)_{w}$.

(2) Obviously, $\left(X\left(Z_{1} \cap Z_{2}\right)\right)_{w} \subseteq\left(X Z_{1} \cap X Z_{2}\right)_{w}$. Conversly, because $X$ is $w$ invertible, we have $\left(X Z_{1} \cap X Z_{2}\right)_{w}=\left(X X^{-1}\left(X Z_{1} \cap X Z_{2}\right)\right)_{w} \subseteq\left(X\left(X^{-1} X Z_{1} \cap\right.\right.$ $\left.\left.X^{-1} X Z_{2}\right)\right)_{w} \subseteq\left(X\left(Z_{1} \cap Z_{2}\right)\right)_{w}$.

Proposition 2.9. Let $D$ be a $w$-sharp domain. If $X, Y$ are nonzero ideals of $D$ such that $(X+Y)_{v}=D$, then $\left(X_{v}+Y_{v}\right)_{w}=D$.

Proof. Let $K$ be the quotient field of $D$. Changing $X$ by $X_{v}$ and $Y$ by $Y_{v}$, we may assume that $X, Y$ are $w$-invertible $v$-ideals, cf. Proposition 2.6. Since $(X+Y)^{2} \subseteq X^{2}+Y$ and $D$ is $w$-sharp, there exist two nonzero ideals $Y_{1}, Y_{2}$ such that $\left(X^{2}+Y\right)_{w}=\left(Y_{1} Y_{2}\right)_{w}$ and $X+Y \subseteq\left(Y_{1}\right)_{w} \cap\left(Y_{2}\right)_{w}$. We claim that $\left(X^{2}+Y\right)_{w}: X=(X+Y)_{w}$. To prove our claim, we perform the following step-by-step computation. First, $\left(X^{2}+Y\right)_{w}: X=\left(\left(X^{2}+Y\right)_{w}: k X\right) \cap D=$ $\left(\left(X^{2}+Y\right) X^{-1}\right)_{w} \cap D=\left(X+Y X^{-1}\right)_{w} \cap D$ because $X$ is $w$-invertible. As $w$ is stable star operation, we get $\left(X+Y X^{-1} \cap D\right)=\left(\left(X+Y X^{-1}\right) \cap D\right)_{w}=$ $\left(X+\left(Y X^{-1} \cap D\right)\right)_{w}$ by modular distributivity. Since $X$ is $w$-invertible, we get $\left(X+\left(Y X^{-1} \cap D\right)\right)_{w}=\left(X+X^{-1}(Y \cap X)\right)_{w}$, cf. Lemma 2.8. Using the fact that $X$ is $w$-invertible (hence $v$-invertible) and Lemma 2.8 , we derive that $\left(X+X^{-1}(Y \cap\right.$ $X))_{w} \subseteq\left(X+X^{-1}(X Y)_{v}\right)_{w} \subseteq\left(X+\left(X X^{-1} Y\right)_{v}\right)_{w}=\left(X+(Y)_{v}\right)_{w}=(X+Y)_{w}$. Putting all these facts together, we get $\left(X^{2}+Y\right)_{w}: X \subseteq(X+Y)_{w}$ and the other inclusion is clear. So the claim is proved. From $\left(X^{2}+Y\right)_{w}=\left(Y_{1} Y_{2}\right)_{w}$, we get $\left(Y_{1}\right)_{w} \subseteq\left(X^{2}+Y\right)_{w}:\left(Y_{2}\right)_{w} \subseteq\left(X^{2}+Y\right)_{w}: X=(X+Y)_{w}$, so $\left(Y_{1}\right)_{w}=(X+Y)_{w}$. Similarly we get $\left(Y_{2}\right)_{w}=(X+Y)_{w}$, hence $\left(X^{2}+Y\right)_{w}=\left((X+Y)^{2}\right)_{w}$. It follows that $(Y)_{w} \subseteq\left(X^{2}+Y\right)_{w}=\left((X+Y)^{2}\right)_{w} \subseteq\left((Y)^{2}+X\right)_{w}$. So $Y_{w}=Y_{w} \cap\left((Y)^{2}+\right.$ $X)_{w}=\left(Y \cap\left((Y)^{2}+X\right)\right)_{w}=\left((Y)^{2}+(Y \cap X)\right)_{w}$ where we used the fact that $w$ is stable star operation and the modular distributivity. By the Lemma 2.8, we have $(X \cap Y) \subseteq(X Y)_{v}$, so we get $(Y)_{w}=\left((Y)^{2}+(Y \cap X)\right)_{w} \subseteq\left((Y)^{2}+(X Y)_{v}\right)_{w}$. Since $Y$ is $w$-invertible, we have $D=\left(Y(Y)^{-1}\right)_{w} \subseteq\left(\left((Y)^{2}+(X Y)_{v}\right)\left(Y^{-1}\right)\right)_{w} \subseteq$ $\left(Y+X_{v}\right)_{w}=(Y+X)_{w}$. Thus $(X+Y)_{w}=D$.

Proposition 2.10. Let $D$ be a $w$-sharp domain. Then every finitely generated nonzero ideal of $D$ is $w$-invertible.

Proof. Let $r, s \in D-\{0\}$. By Proposition 2.6, the ideal $X=(r D+s D)_{v}$ is $w$-invertible. (hence $v$-invertible), So $\left(r X^{-1}+s X^{-1}\right)_{v}=D$. By Proposition 2.9, we get $\left(r X^{-1}+s X^{-1}\right)_{w}=D$. Hence $X=\left(\left(r X^{-1}+s X^{-1}\right) X\right)_{w}=(r D+s D)_{w}$ 
because $X$ is $w$-invertible. Thus every two-generated nonzero ideal of $D$ is $w$ invertible. Now the proof of [15, Proposition 22.2] can be easily adapted to show that every finitely generated nonzero ideal of $D$ is $w$-invertible.

Let $D$ be a domain with quotient field $K$. According to [6], a family $\mathcal{F}$ of nonzero prime ideals of $D$ is called independent of finite character family (IFC family), If (a) $D=\cap_{P \in \mathcal{F}} D_{P}$ (b) every nonzero $d \in D$ belongs to only finitely many members of $\mathcal{F}$ and $(c)$ every nonzero prime ideal of $D$ is contained in at most one member of $\mathcal{F}$.

Proposition 2.11. Let $D$ be a domain. Assume that (1) every $r \in D-\{0\}$ is contained in only finitely many maximal w-ideals, and (2) for every $Q \in \operatorname{Max}_{w} D, D_{Q}$ is a valuation domain with value group a complete subgroup of the reals.

Then $D$ is a $w$-sharp domain.

Proof. By [16], $D=\cap_{Q} D_{Q}$ where $Q$ runs in the set of maximal $w$-ideals. As each $D_{Q}$ is a valuation domain with value group a complete subgroup of the reals, every $Q$ has height one. It follows that $\operatorname{Max}_{w}(D)$ is an IFC family. We show that $D$ is $w$-sharp. Let $X, Y_{1}, Y_{2}$ be nonzero ideals of $D$ such that $X \supseteq$ $Y_{1} Y_{2}$. Let $L_{1}, \ldots L_{n}$ be the maximal $w$-ideals of $D$ containing $Y_{1} Y_{2}$. Since $D_{L_{i}}$ is sharp, there exist $A_{i}, B_{i}$ ideals of $D_{L_{i}}$ such that $X D_{L_{i}}=A_{i} B_{i}, A_{i} \supseteq Y_{1} D_{L_{i}}$ and $B_{i} \supseteq Y_{2} D_{L_{i}}$ for all $i$ between 1 and $n$. Set $A_{i}^{\prime}=A_{i} \cap D, B_{i}^{\prime}=B_{i} \cap D$, $i=1,2 \ldots . n, A=A_{1}^{\prime} \ldots A_{n}^{\prime}$ and $B=B_{1}^{\prime} \ldots B_{n}^{\prime}$. By [6, Lemma 2.3], $L_{i}$ is the only element of $\operatorname{Max}_{w}(D)$ containing $A_{i}^{\prime}\left(\right.$ resp. $\left.B_{i}^{\prime}\right)$, thus it can be checked that $X D_{L}=(A B) D_{L}, A D_{L} \supseteq Y_{1} D_{L}$ and $B D_{L} \supseteq Y_{2} D_{L}$ for each $L \in \operatorname{Max}_{w}(D)$. So, we have $X_{w}=(A B)_{w}, A_{w} \supseteq Y_{1}$ and $B_{w} \supseteq Y_{2}$. Consequently, $D$ is $w$-sharp.

Proposition 2.12. Let $D$ be a countable domain such that $D$ is a PwMD and $X_{v}$ is $w$-invertible for each nonzero ideal $X$ of $D$. Then every nonzero element of $D$ is contained in only finitely many maximal $w$-ideals.

Proof. Let us deny. By [13, Corollary 5], there exists a nonzero element $r$ and an infinite family $\left(X_{k}\right)_{k \geq 1}$ of $w$-invertible proper ideals containing $r$ which are mutually $w$-comaximal (i.e., $\left(X_{p}+X_{q}\right)_{w}=D$ for every $\left.p \neq q\right)$. For each nonempty set $\Omega$ of natural numbers, consider the $v$-ideal $X_{\Omega}=\cap_{k \in \Omega} X_{k}$ (note that $r \in X_{\Omega}$ ). By hypothesis, $X_{\Omega}$ is $w$-invertible. We claim that $X_{\Omega} \neq X_{\Omega^{\prime}}$ whenever $\Omega$ and $\Omega^{\prime}$ are distinct nonempty sets of natural numbers. Deny. Then there exists a nonempty set of natural numbers $\Delta$ and some $l \notin \Delta$ such that $X_{l} \supseteq X_{\Delta}$. Consider the ideal $A=\left(X_{l}^{-1} X_{\Delta}\right)_{w} \supseteq X_{\Delta}$. If $q \in \Delta$, then $X_{q} \supseteq X_{\Delta}=\left(X_{l} A\right)_{w}$, so $X_{q} \supseteq A$, because $\left(X_{q}+X_{l}\right)_{w}=D$. It follows that 
$X_{\Delta} \supseteq A$, so $X_{\Delta}=A=\left(X_{l}^{-1} X_{\Delta}\right)_{w}$. Since $X_{\Delta}$ is $w$-invertible, we get $X_{l}=D$, a contradiction. Thus the claim is proved. But then it follows that $\left\{X_{\Omega} \mid \emptyset \neq \Omega \subseteq\right.$ $\mathbb{N}\}$ is an uncountable set of $w$-invertible ideals. This leads to a contradiction, because $D$ being countable, it has countably many $w$-ideals of finite type.

Corollary 2.13. Let $D$ be a countable domain such that $D$ is $w$-sharp. Then $D$ is a $w$-Dedekind domain.

Proof. We may assume that $D$ is not a field. By Proposition 2.4, $D$ is a PwMD. Now Propositions 2.6 and 2.12 show that every nonzero element of $D$ is contained in only finitely many maximal $w$-ideals. Let $Q$ be a maximal $w$-ideal of $D$. By Proposition 2.4, $D_{Q}$ is a countable valuation domain with value group $\mathbb{Z}$ or $\mathbb{R}$, so $D_{Q}$ is a DVR. Thus $D$ is a $w$-Dedekind domain, cf. [7, Theorem 4.11].

Acknowledgements. We would like to express our sincere thanks to The University of Lahore, Pakpattan Campus, Pakpattan for their support in this research work.

\section{References}

[1] Z. Ahmed, T. Dumitrescu, Almost quasi-Schreier domains, Comm. Algebra, 41 (2013), 1685-1696, doi: 10.1080/00927872.2011.649505.

[2] Z. Ahmad, T. Dumitrescu, M. Epure, A Schreier domain type condition, Bull. Math. Soc. Sci. Math. Roumanie Tome, 55(103) (2012), 241-247.

[3] D.D. Anderson, Star-operations induced by overrings, Comm. Algebra, 16 (1988), 25352553, doi: 10.1080/00927879808823702.

[4] D.D. Anderson, S.J. Cook, Two star-operations and their induced latices, Comm. Algebra, 28 (2000), 2461-2475, doi: 10.1080/00927870008826970.

[5] D.D. Anderson, T. Dumitrescu, M. Zafrullah, Quasi-Schreier domains II, Comm. Algebra, 35 (2007), 2096-2104, doi: 10.1080/00927870701302107.

[6] D.D. Anderson,M. Zafrullah, Independent locally-finite intersections of localizations, Houston J. Math., 25 (1999), 433-452.

[7] S.El Baghdadi, M. Fontana, G. Picozza, Semistar Dedekind domains, J. Pure Appl. Algebra, 193 (2004), 27-60, doi: 10.1016/j.jpaa.2004.03.011.

[8] A. Bouvier, M. Zafrullah, On some class groups of an integral domain, Bull. Soc. Math. Grèce, 29 (1988), 45-59.

[9] P.M. Cohn, Bézout rings and their subrings, Proc. Cambridge Philos. Soc., 64 (1968), 251-264.

[10] T. Dumitrescu, W. Khalid, Almost-Schreier domains, Comm. Algebra, 38 (2010), 29812991, doi: 10.1080/00927870903100101. 
[11] T. Dumitrescu, R. Moldovan, Quasi-Schreier domains, Math. Reports, 5 (2003), 121-126.

[12] T. Dumitrescu, M. Zafrullah, Characterizing domains of finite *-character, J. Pure Appl. Algebra, 214 (2010), 2087-2091, doi: 10.1016/j.jpaa.2010.02.014.

[13] T. Dumitrescu, M. Zafrullah, t-Schreier domains, Comm. Algebra, 39 (2011), 808-818, doi: $10.1080 / 00927871003597642$.

[14] M. Fontana, P. Jara, E. Santos, Prüfer $\star$-multiplication domains and semistar operations, J. Algebra Appl., 2 (2003), 21-50, doi: 10.1142/S0219498803000349.

[15] R. Gilmer, Multiplicative Ideal Theory, Pure Appl. Math., No. 12, Marcel Dekker, Inc, New York, 1972.

[16] M. Griffin, Some results on v-multiplication rings, Canad. J. Math., 19 (1967), 710-722, doi: 10.4153/CJM-1967-065-8. Canad. J. Math. 19(1967), 710-722

[17] B.G. Kang, Prüfer $v$-multiplication domains and the ring $R[x]_{N_{v}}, J$. Algebra, 123 (1989), 151-170, doi: 10.1016/0021-8693(89)90040-9.

[18] I. Kaplansky, Commutative Rings, The University of Chicago Press, Chicago, 1974.

[19] S. McAdam, D.E. Rush, Schreier Rings, Bull. London Math. Soc., 10 (1978), 77-80, doi: 10.1112/blms/10.1.77.

[20] M. Zafrullah, On a property of pre-Schreier domains, Comm. Algebra, 15 (1987), 18951920, doi: 10.1080/00927878708823512. 